\title{
MULTIPLEX POLYMERASE CHAIN REACTION (PCR) FOR THE DETECTION OF NEISSERIA GONORRHOEAE AND THE QUINOLONE RESISTANCE GENE IN PAKISTAN
}

\author{
Hafsa Waseem, Afnan Naeem, Sakhawat Ali, Sharjeel Sarfraz, Javaid Usman, Mehreen Gilani \\ Army Medical College/National University of Medical Sciences (NUMS) Rawalpindi Pakistan
}

\begin{abstract}
Objective: To detect Neisseria gonorrhoeae from the urine of male patients reporting with active urethral discharge using multiplex polymerase chain reaction (PCR). And the simultaneous detection of the quinolone resistance determining region (QRDR) on the Neisseria gonorrhoeae gene using multiplex polymerase chain reaction.

Study Design: Cross sectional study.

Place and Duration of Study: Microbiology department, Army Medical College Rawalpindi Pakistan, from Mar to Dec 2018. Methodology: Male patients with active urethral discharge with no past history of antibiotic use for urethral discharge were included in study and patients without active urethral discharge and history of antibiotic use for urethral discharge were excluded. Urine of patients of active urethral discharge was collected and multiplex polymerase chain reaction was done by using two forward primers along with common reverse primer.

Results: In this study 24 (40\%) of patients who presented with active urethral discharge were positive for gonorrhea. However Quinolone Resistance Determining Region is detected in 17 (70.83\%) of cases and only 7 (29.17\%) were sensitive to ciprofloxacin.

Conclusion: The multiplex polymerase chain reaction is very efficient and effective method for the simultaneous detection of Neisseria gonorrhoeae and status of isolate susceptibility to ciprofloxacin. And in Pakistan ciprofloxacin cannot be used as first line drug for the treatment of gonorrhea.
\end{abstract}

Keywords: Gonorrhea, Multiplex polymerase chain reaction, Quinolone resistance determining region.

This is an Open Access article distributed under the terms of the Creative Commons Attribution License (https://creativecommons.org/licenses/by-nc/4.0/), which permits unrestricted use, distribution, and reproduction in any medium, provided the original work is properly cited.

\section{INTRODUCTION}

Gonorrhea is the second most prevalent sexually transmitted bacterial disease caused by bacteria Neisseria gonorrhoeae ${ }^{1}$. The center for disease control (CDC) estimates that approximately 820,000 new gonococcal infections occur in United States each year, and out of these 820.000 only half are detected and reported to $\mathrm{CDC}^{2}$.

Neisseria gonorrhoeae causes urethritis in males and cervicitis in females. More than half of gonococcus infected females and a small number of infected males are asymptomatic. But if urogenital gonorrhea remain untreated it ascends and causes serious complications like pelvic inflammatory disease, ectopic pregnancy in females and epididymitis and penile edema in males. It also encourages transmission of HIV and other sexually transmitted infections.

To control gonococcal infections the cornerstone is effective treatment with appropriate antimicrobials to treat the case and to prevent its further transmission as there is no vaccine available against gonorrhea. And the ability of Neisseria gonorrhoeae to develop resistance against antimicrobials making it complicated. It

Correspondence: Dr Hafsa Waseem, Flat No. 101, Sector E-10, Defense Complex, Islamabad Pakistan

Received: 01 Jul 2020; revised received: 22 Oct 2020; accepted: 04 Nov 2020 has developed resistance to each and every class of antimicrobial offered for treatment ${ }^{3}$.

In USA and UK dual therapy regimen of ceftriaxone and azithromycin is recommended for uncomplicated gonococcal infections. But the history of Neisseria gonorrhoeae tells us that it develops antimicrobial resistance against every drug offered for treatment so this dual therapy only seems some delay rather than a permanent solution, Therefore urgent action is needed to combat this problem. Currently one of the limitations to control the antimicrobial resistance problem of Neisseria gonorrhoeae is lack of data so surveillance data need to be enhanced and implemented.

Gram stained smear is reliable, inexpensive and a fast method for detection of Neisseria gonorrhoeae but it is only reliable when patients with active urethral discharge were selected for the study. In well developed countries nucleic acid amplification test (NAAT) is commonly used for diagnosis of gonorrhea. NAAT is recommended because it has high sensitivity as compared to culture, ease of sample collection and sample transport as it can also detect gonorrhea in first void urine sample and endocervical swabs ${ }^{4}$. A molecular method for the simultaneous detection of Neisseria gonorrhoeae and its ciprofloxacin susceptibility status has been described. To include most conserved region 
in gyrA sequence that is not common in non-Neisseria gonorrhoeae species the primer gyrA-W forward was designed. To include gyrA sequence harboring S91 and D95 mutations the gyrA-M forward primer was designed. These primers paired with common reverse primer.

The genomic regions of Neisseria gonorrhoeae gyrA and parC are associated with ciprofloxacin resistance. ParC mutations alone is not associated with ciprofloxacin resistance and gyrA mutation alone is associated with low level resistance. However gyrA mutation along with parC mutations maintains higher levels of resistance. About 145 studies from different countries including Switzerland, Canada, South Africa and Brazil support that gyrA mutation alone is sufficient to confirm ciprofloxacin susceptibility ${ }^{5}$. So in this study we identified ciprofloxacin resistant and sensitive isolates only by considering gyrA. However many real time polymerase chain reaction based studies were conducted on clinical samples in which only mutations at the gyrA S91 locus were detected ${ }^{6,7}$. But studies showed that ciprofloxacin intermediate resistance mainly due to mutations in either S91 or D95 loci while ciprofloxacin resistance arose due to mutations in both $\mathrm{S} 91$ and D95 loci $^{8}$. So in this study we consider mutations both at S91 and D95 loci.

In 2013 in the United Kingdom 70\% isolates of gonorrhea were susceptible to ciprofloxacin 9 and in the United States $74.1 \%$ isolates of Neisseria gonorrhoeae showed susceptibility to ciprofloxacin ${ }^{10}$. So prescribing ceftriaxone to all patients is a poor strategy. We should identify ciprofloxacin sensitive strains so that unnecessary ceftriaxone can be avoided and be used only for ciprofloxacin resistant isolates.

In Pakistan people are reluctant to seek medical advice due to lack of facilities, cultural constraints and taboo. This study has the potential of rapid diagnosis of Neisseria gonorrhoeae and its susceptibility to ciprofloxacin which leads to early diagnosis and timely management.

\section{METHODOLOGY}

It was a cross sectional study and the study was carried out at the department of Microbiology, Army Medical College Rawalpindi Pakistan, from March 2018 to December 2018.

The sample size was calculated by using WHO calculator. Confidence level 95 Anticipated population proportion $p=0.04^{11}$. Absolute precision required $d=$ 0.05 and the minimum sample size $(n=60)$ and the sampling technique was non probability consecutive sampling technique.

All males' first time attending skin and urology department with complaint of active urethral discharge with no previous history of antibiotic use for urethral discharge were included in the study and patients without active urethral discharge and history of antibiotic use for urethral discharge were excluded from study.

After getting approval from Ethics Review Committee Army Medical College Rawalpindi samples were collected from the department of Dermatology Pak Emirates Military Hospital Rawalpindi and department of urology Benazir Bhutto Hospital Rawalpindi. Urine samples of 60 male patients with active urethral discharge were collected insterile plastic container and transported to laboratory within one hour of collection and stored at $4^{\circ} \mathrm{C}$. DNA was extracted from the urine sample of each individual using commercially available kit. (PureLink Microbiome DNA purification kit Cat\# A29790 M/s Invitrogen).

Forward primer gyrA-W included the most conserved region in gyrA sequence that is not common in non-Neisseria gonorrhoeae species (5"GCGATTCCGC AGTTTACGA3") was used. The gyrA-M forward primer included the gyrA sequence harboring $S 91$ and D95 mutations (5"TACCACCCCCACGGCGATTT3") which was used for quinolone resistance determination. These primer was paired with common reverse primer gyrA-R (5"5CGAAATTTTGCGCCATACGGA CGAT3"). For detecting Neisseria gonorrhoeae and its ciprofloxacin status in a single amplification these primers in multiplex format has $100 \%$ sensitivity and specificity ${ }^{8}$.

Real time polymerase chain reaction (RT-PCR) was performed using Cepheid smart cycler on a 16 well platform with the Maxima SYBR Green/ROX q PCR Master Mix (2x) (Cat \#K0221 M/s Thermo Scientific). PCR reactions contained $0.5 \mu$ of each primer $(10 \mu \mathrm{M}), 2 \mu \mathrm{l}$ of DNA template $(50 \mathrm{ng} / \mu \mathrm{l})$ and $5 \mu \mathrm{l}$ of $2 \times$ SYBR Green and the final reaction volume was adjusted with deionized water to $10 \mu 1$. According to manufacturer guidelines PCR was conducted with the following modifications: activation and initial holding at $50^{\circ} \mathrm{C}$ for 2 minutes than secondary holding at $95^{\circ} \mathrm{C}$ for 2 minutes. PCR was performed with positive and negative controls for 25 cycles at $95^{\circ} \mathrm{C}$ for 15 seconds and $60^{\circ} \mathrm{C}$ for 30 seconds along with positive and negative controls. Post PCR melt curve was performed with $0.3^{\circ} \mathrm{C}$ temperature increments between $60-95^{\circ} \mathrm{C}$. Data 
were collected at annealing stage (amplification data) and then at melting stage. Amplification till 25 cycles were taken as positive for Neisseria gonorrhoeae and melt curve $78^{\circ} \mathrm{C}$ were taken as ciprofloxacin sensitive and melt curve values $80-80.3^{\circ} \mathrm{C}$ were taken as ciprofloxacin resistant ${ }^{8}$.

Data was analyzed by using SPSS-22. For qualitative variables percentages and frequency were calculated. Quantitative values were expressed as mean \pm standard deviation.

\section{RESULTS}

In our study the minimum age was 22 years and the maximum age was 38 years Mean age of patients positive for Neisseria gonorrhoeae was $26.4 \pm 3.11$ years and mean age of patients negative for Neisseria gonorrhoeae was $27.7 \pm 3.98$ years.

Out of the total, $21(35 \%)$ were from patients in the age group 22-25 years, $26(43.3 \%)$ were from patients in the age group 26-29 years, $7(11.6 \%)$ were from patients in the age group 30-33 years and $6(10 \%)$ were from patients in the age group with 34 years and above. A total of 60 patients were included in this study out of them 24 samples $(40 \%)$ were positive for Neisseria gonorrhoeae and $36(60 \%)$ were negative for Neisseria gonorrhoeae.

Out of total 24 positive patients for Neisseria gonorrhoeae, 9 (37.5\%) were from age group 22-25 years, 11 (45.8\%) were from age group 26-29 years, 3 $(12.5 \%)$ were from age group $30-33$ years and $1(4.1 \%)$ were from age group 34 years and above.

Out of total 24 positive samples of Neisseria gonorrhoeae $17(70.83 \%)$ samples were positive for Quinolone Resistance Determining Region (QRDR) and 7 (29.17\%) were negative for QRDR as shown in table-II.

Table-I: Frequency of Neisseria gonorrhoeae in urethral discharge $(n=60)$.

\begin{tabular}{l|c}
\hline Samples & n (\%) \\
\hline Positive & $24(40 \%)$ \\
\hline Negative & $36(60 \%)$ \\
\hline Total & $60(100 \%)$ \\
\hline $\begin{array}{l}\text { Table-II: Frequency of quinolone resistance determining } \\
\text { region (QRDR) (n=24). }\end{array}$ \\
\hline Sample & $\mathbf{n ~ ( \% )}$ \\
\hline Positive & $17(70.83 \%)$ \\
\hline Negative & $7(29.17 \%)$ \\
\hline Total & $24(100 \%)$ \\
\hline
\end{tabular}

\section{DISCUSSION}

To my knowledge in Pakistan this is the first report of simultaneous detection of Neisseria gonorrho- eae and its ciprofloxacin susceptibility status in a single test that is rapid, inexpensive and $100 \%$ sensitive and specific.

In our study $40 \%$ patients of active urethral discharge were positive for Neisseria gonorrhoeae which is comparable to the studies done on this topic in Pakistan, Kuwait and Dhaka ${ }^{12-14}$. In these studies $27.5 \%, 31.5 \%$ and $30.27 \%$ patients with active urethral discharge were positive for Neisseria gonorrhoeae respectively. However rate is higher when compared with studies conducted in South Africa and India ${ }^{15,16}$. In these studies multiplex PCR was performed $85 \%$ and $76.8 \%$ patients with urethritis were positive for Neisseria gonorrhoeae respectively.

In current study $70.83 \%$ isolates of Neisseria gonorrhoeae were resistant to ciprofloxacin. Before that a study was conducted in Pakistan in 2012-2014 at the Aga Khan University Karachi, in which Neisseria gonorrhoeae showed $86 \%$ resistance to ciprofloxacin ${ }^{17}$.

Study conducted from 2007 to 2011 in Pakistan, India and Bhutan on genetic characteristic and antimicrobial susceptibility of Neisseria gonorrhoeae and result showed more resistance to ciprofloxacin than our study it showed that not a single isolate of Neisseria gonorrhoeae was susceptible to ciprofloxacin, $94 \%$ were resistant to ciprofloxacin and $6 \%$ were intermediately susceptible and all isolates showed mutations in Quinolone Resistance Determining Region at gyrA and parC genes ${ }^{18}$.

A study was conducted in India on the antimicrobial resistance of Neisseria gonorrhoeae from 2015 to 2017 which showed Neisseria gonorrhoeae was 100\% resistant to quinolones and gyrA and parC were observed in all isolates of quinolone resistant Neisseria gonorrhoeae $^{19}$. As compared to our region in the United States and the United Kingdom resistance of Neisseria gonorrhoeae to ciprofloxacin is very low.

Study in United States showed 25.9\% ciprofloxacin resistance to Neisseria gonorrhoeae and according to CDC $19.2 \%$ ciprofloxacin resistance among gonococcal strains were reported ${ }^{9}$. In United Kingdom study reported $30 \%$ cases of gonorrhea were resistant to ciprofloxacin ${ }^{20}$.

Studies carried out over the world on susceptibility of Neisseria gonorrhoeae to ciprofloxacin shows marked disparity of results in different regions of world so appropriate antimicrobial treatment according to susceptibility pattern in your region and according to case is essential. 


\section{Funding Source}

It is funded by National Medical University (NUMS) and Department of Pathology Army Medical College Rawalpindi.

\section{CONCLUSION}

This study showed that Neisseria gonorrhoeae was positive in the urine of $40 \%$ patients of active urethral discharge and $70.83 \%$ Neisseria gonorrhoeae showed resistance to quinolone. So in Pakistan ciprofloxacin cannot be used as first line drug for the treatment of gonorrhea and we should use internationally recommended, ceftriaxone and azithromycin as first line drugs for the treatment of gonorrhea.

\section{CONFLICT OF INTEREST}

This study has no conflict of interest to be declared by any author.

\section{REFERENCES}

1. Unemo M, Shafer WM. Antibiotic resistance in Neisseria gonorrhoeae: origin, evolution, and lessons learned for the future. Ann N Y Acad Sci 2011; 1230(1): E19-28.

2. Satterwhite CL, Torrone E, Meites E, Dunne EF, Mahajan R, Ocfemia MCB, et al. Sexually transmitted infections among US women and men: prevalence and incidence estimates, 2008. Sex Transm Dis 2013; 40(3): 187-93.

3. Anschuetz G, Asbel L, Salmon ME, Johnson CC. Use of first-line treatment for Neisseria gonorrhoeae after treatment guideline changes. Sex Transm Dis 2014; 41(1): 64-66.

4. Low N, Unemo M, Jensen JS, Breuer J, Stephenson JM. Molecular diagnostics for gonorrhoea: implications for antimicrobial resistance and the threat of untreatable gonorrhoea. PLoS Med 2014; 11(2): e1001598-03.

5. Allan-Blitz LT, Hemarajata P, Humphries RM, Kimble M, Elias S, Klausner JD. Ciprofloxacin May be Efficacious in Treating WildType Gyrase A Genotype Neisseria gonorrhoeae Infections. Sex Transm Dis 2018; 45(4): e18-20.

6. Buckley C, Trembizki E, Donovan B, Chen M, Freeman K, Guy $\mathrm{R}$, et al. A real-time PCR assay for direct characterization of the Neisseria gonorrhoeae GyrA 91 locus associated with ciprofloxacin susceptibility. J Antimicrob Chemother 2016; 71(2): 353-56.

7. Allan-Blitz LT, Wang X, Klausner JD. Wild-Type gyrase a genotype of neisseria gonorrhoeae predicts in vitro susceptibility to ciprofloxacin: a systematic review of the literature and metaanalysis. Sex Transm Dis 2017; 44(5): 261-65.
8. Perera SR, Khan NH, Martin I, Taheri A, Parti RP, Levett PN, et al. Multiplex Real-Time PCR Assay for Simultaneous Identification of Neisseria gonorrhoeae and Its Ciprofloxacin Susceptibility Status. J Clin Microbiol 2017; 55(11): 3201-209.

9. Hemarajata P, Yang S, Soge O, Humphries R, Klausner J. Performance and verification of a real-time PCR assay targeting gyr A gene for prediction of ciprofloxacin resistance in Neisseria gonorrhoeae. J Clin Microbiol 2016: 54(3); 03032-15.

10. Low N, Unemo M. Molecular tests for the detection of antimicrobial resistant Neisseria gonorrhoeae: when, where, and how to use. Curr Opin Infect Dis 2016; 29(1): 45-51.

11. Nirmal K, Saha R, Ramachandran V, Das S, Bhattacharya S. Resistance in Neisseria gonorrhoeae: A Cause of Concern. Int J Curr Microbiol App Sci 2016; 5(11): 140-44.

12. Rehan N. Profile of men suffering from sexually transmitted infections in Pakistan. J Pak Med Assoc 2006; 56(Suppl-1): S60-65.

13. Al Mutairi N, Joshi A, Nour Eldin O, Sharma AK, El Adawy I, Rijhwani M. Clinical patterns of sexually transmitted diseases, associated sociodemographic characteristics, and sexual practices in the Farwaniya region of Kuwait. Int J Dermatol 2007; 46(6): 594-99.

14. Jahan F, Shamsuzzaman S, Akter S. Diagnosis of common bacterial causes of urethritis in men by Gram stain, culture and multiplex PCR. Malays J Pathol 2014; 36(3): 175-80.

15. Mhlongo S, Magooa P, Müller EE, Nel N, Radebe F, Wasserman E, et al. Etiology and STI/HIV coinfections among patients with urethral and vaginal discharge syndromes in South Africa. Sex Transm Dis 2010; 37(9): 566-70.

16. Bala M, Ray K, Gupta S, Muralidhar S, Jain R. Changing trends of antimicrobial susceptibility patterns of Neisseria gonorrhoeae in India and the emergence of ceftriaxone less susceptible $\mathrm{N}$. gonorrhoeae strains. J Antimicrob Chemother 2007; 60(3): 582-86.

17. Jabeen K, Bhawan Mal P, Khan E, Chandio S, Jacobsson S, Unemo M. Antimicrobial resistance and Neisseria gonorrhoeae multiantigen sequence typing (NG-MAST) genotypes in $\mathrm{N}$. gonorrhoeae during 2012-2014 in Karachi, Pakistan. BMC Infect Dis 2016; 16(1): 353-55.

18. Sethi S, Golparian D, Bala M, Dorji D, Ibrahim M, Jabeen K, et al. Antimicrobial susceptibility and genetic characteristics of Neisseria gonorrhoeae isolates from India, Pakistan and Bhutan in 2007-2011. BMC Infect Dis 2013; 13(1): 35-38.

19. Sharma M, Rizvi M, Gupta R, Azam M, Khan HM, Parvez A. Alarming resistance of Neisseria gonorrhoeae in a tertiary care hospital of North India. Ind J Med Microbiol 2018; 36(2): 285-88.

20. Turner KM, Christensen H, Adams EJ, McAdams D, Fifer H, McDonnell A, et al. Analysis of the potential for point-of-care test to enable individualised treatment of infections caused by antimicrobial-resistant and susceptible strains of Neisseria gonorrhoeae: a modelling study. BMJ Open 2017; 7(6): e015447-50. 